\title{
Research Paper \\ Construction and Validation of a Scale of Research Anxiety for Students
}

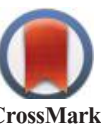

\author{
Fatemeh Gholami Booreng ${ }^{1}$, Behrooz Mahram ${ }^{2 *}$, Hossein Kareshki ${ }^{3}$
}

1. MA in Educational Research, Department of Education, Faculty of Education and Psychology, Ferdowsi University of Mashhad, Mashhad, Iran.

2. PhD in Curriculum Studies, Associate Professor, Department of Education, Faculty of Education and Psychology, Ferdowsi University of Mashhad, Mashhad, Iran.

3. PhD in Educational Psychology, Associate Professor, Department of Education, Faculty of Education and Psychology, Ferdowsi University of Mashhad, Mashhad, Iran.

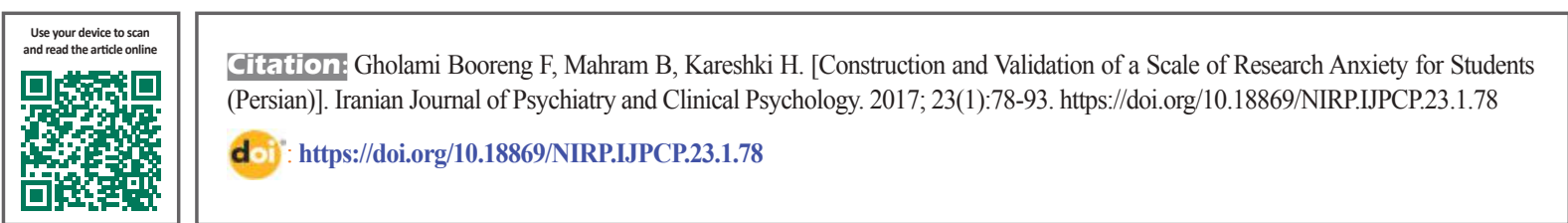

Received: 16 Sep. 2016

Accepted: 04 Jan. 2017

Key words:

Research anxiety,

Student, Validation

\section{A B STRACT}

Objectives The aim of this study was to design and validate a scale of research anxiety for students at the Ferdowsi University of Mashhad.

Methods The correlation method was used in this study. The population consisted of MA students at the Ferdowsi University of Mashhad in the academic year 2014-15. The prepared scales were analyzed in two stages: preliminary and final stages. In the preliminary stage, the scale was implemented on 100 students selected via convenient sampling, and in the final stage, the tool was implemented on 400 students selected via quota sampling method based on gender and field of study. Data were analyzed using SPSS software.

Results The results showed that the factor analysis using varimax rotation supported only one factor, and 40 items were selected on each phrase for at least 0.3 loadings. The validity of the scale was obtained with the help of the judgment of experts, convergent-divergent validity, and factor analysis. Scale converging-diverging validity done of correlation with the Spielberger State-Trait anxiety (STAI) and Cooper Smith self-esteem. The results showed negative relation (-63.0) between self-esteem and research anxiety and a strong positive correlation (0.650) between Spielberger State-Trait Anxiety and research anxiety. Scale reliability was 0.87 via Cronbach's alpha and 0.76 via re-test after seven days.

Conclusion The results showed that research anxiety scale has acceptable reliability and validity and can be used for a variety of applications.

\section{Extended Abstract}

\section{Introduction}

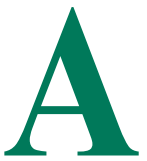

nxiety refers to a condition where a person is extremely worried, stressed, and upset at the time of a terrible event $[1,2]$. Anxiety research comprises individual factors affecting the performance of researchers.
The study of its structural aspects (i.e., components) and their interconnections could contribute to the organized knowledge in this area and promoting research level students. Cultural factors can dramatically affect in the research tools Given that there is a lack of useful tools in the field of research anxiety of students, this study aimed to identify the factors important for the anxiety research. It also aimed at designing a questionnaire as a construction tool to measure the factors among the students. In other words, the purpose of

* Corresponding Author:

Behrooz Mahram, PhD

Address: Department of Educational Sciences, Faculty of Education and Psychology, Ferdowsi University of Mashhad, Mashhad, Iran.

Tel: +98 (513) 8805878

E-mail: bmahram@um.ac.ir 
this study was to investigate the factor structure of research anxiety scale and its psychometric properties.

\section{Method}

In the current study, the correlational research method was used. The population comprised of all graduate students of the Ferdowsi University of Mashhad who were enrolled in the academic year of 2014-15, i.e., 1031 women and 843 men. The other inclusion criterion was not defending their dissertation at the time of this study. The prepared research anxiety scale was implemented in both the preliminary and final stages. In the preliminary stage, the tool was implemented on 100 students selected with convenient sampling. In the final stage, the tool was implemented on over 400 students selected with quota sampling based on gender and field of study. Data were analyzed by factor analysis using statistical software SPSS version 20. The validity of the scale was confirmed by arbitration specialists, convergent-divergent validity and factor analysis. The reliability of the scale was obtained using test-retest and Cronbach's alpha.

\section{Results}

A total of 11 factors could be extracted according to the number of special values higher than one and gravel graph (Figure 1). For better separation and extraction, Varimax rotation was used. According to accounting power less than $5 \%$, only two factors were extracted that accounted for $27.63 \%$ of the total variance. Thus, about $21.72 \%$ of the variance is accounted for only by the first factor. The results also indicated that 34 out of 40 questions were focused on the first factor. The load factor of six questions focused on two factors. Thus, according to the original matrix factor and related weights, the questionnaire was considered in the form of one factor.

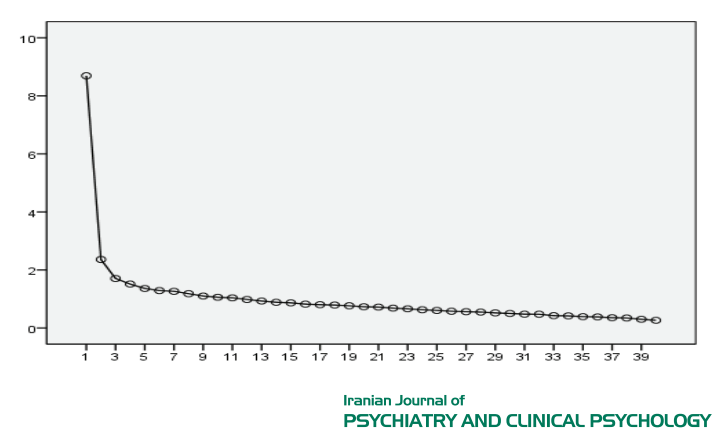

Figure 1. Scree plot for the factors extracted

As seen in Table 1, Bartlett's test is significant; therefore, there is a possibility of conducting factor analysis. To check the validity of the test, factor analysis, professional judgment, and convergent-divergent validity were used. The convergence and divergence of research anxiety scale were investigated using Cooper-Smith Self-Esteem Scale and the Spielberger State-Trait Anxiety Inventory. Fifty-five subjects responded to the Cooper-Smith self-esteem questionnaire and the Spielberger State-Trait Anxiety Inventory. It was found that the correlation of Anxiety scale research with Spiel-Berger was equal to 0.65 and correlation with SelfEsteem Test was -0.63 .

To study the questionnaire validity were used of factor analysis, convergent and divergent validity and judgment of experts. In the judgment of experts were included validity by 5 of Counseling center consultant. The reliability of the instrument through Cronbach's alpha was equal to 0.87 , and its stability using the test-retest method on 22 subjects within one-week interval from the first pilot study was 0.76 . Generally, reliability coefficient of research anxiety test was at the optimal level for the entire sample. The following table shows the calculation of Cronbach's alpha coefficient for the general research anxiety scale.

Table 1. KMO index and Bartlett's test of sphericity for Research Anxiety Scale

\begin{tabular}{cccc}
\hline \multicolumn{4}{c}{ Bartlett Test } \\
\hline Chi square & Degree of freedom & Level of significance & KMO Test \\
\hline 3627.62 & 780 & 0.001 & 0.88 \\
\hline & & & $\begin{array}{c}\text { Iranian Journal of } \\
\text { PSYCHIATRY AND CUNICAL PSYCHOLOGY }\end{array}$
\end{tabular}

Table 2. Cronbach's alpha coefficient for general research anxiety scale

PSYCHIATRY AND CUNICAL PSYCHOLOGY

\begin{tabular}{cc}
\hline Number of items & Cronbach's alpha coefficient \\
\hline 40 & 0.93 \\
\hline
\end{tabular}


According to Table 2, questions have not the same variance and do not enjoy the same status. Cronbach's alpha value after removing each question also shows that removing the statement will not lead to significant increase in reliability and reliability for 40 items after removing the distorted questionnaire, is equal to 0.87 .

\section{Discussion}

Given the high prevalence of research anxiety in the student population, it seems necessary to have a comprehensive scale to measure this disorder. Therefore, the findings are discussed based on research goals. The first objective was to examine the factor structure of research anxiety scale; the factor analysis showed that he items such as (When choosing the subject, I have the pleasant peace of mind), (I do not worry about choosing the appropriate subject for the research study), (For selecting a supervisor, I feel apprehension and doubt), (During talks with the supervisor, I am relaxed), (I think, I feel relief when writing a research project), (In preparing my research plan, I am very relieved), (If I could design my research well, I will feel confidence), (If I plan to defend my research, I will feel relaxed), (In the case of my statistical work, I have no fear and apprehension), (I think my sample is not effective),(I'm sure in preparing my research, I have a good feeling), (I am worried when conducting my statistical analysis work, I suffer from insomnia) loaded no factor after the rotation, and they were excluded as inappropriate items.

The second objective of the research was psychometric examination (validity, reliability, normality) of research anxiety scale. It was found that the questionnaire supports only one factor since 34 questions from 40 questions are focused on the first factor. Also, according to Klein (2001), the best situation in a test is that the test measures only one variable, and to ensure this, the test was conducted with many questions and the correlations between questions were factor analyzed. The questions that had an overall load on the factor were selected for the test. According to the results and theories about self-esteem [58-62], anxiety and stress may be associated with different forms of self-esteem. Therefore, in this research, the relationship of this variable with self-esteem was evaluated as an indicator of validity. The correlation coefficient suggests that the more is the research anxiety, the lower will be the self-esteem in students. Also according to studies in the field of anxiety [63-65] and the relationship of anxiety with each other [66], the Spielberger State-Trait Inventory has a significant positive correlation with research anxiety; this correlation is also confirmed in our study confirms.

To investigate the norm, the $t$ and $z$ equivalent scores of raw scores on the anxiety scale were investigated. By con- verting the raw scores to percentile ranks, $\mathrm{Z}$ score and $\mathrm{T}$ scores, it was possible to interpret the test scores of research anxiety. Generally, anxiety refers a complex combination of negative emotional reactions such as anxiety, fear, and panic [3]. The results of this study can be used to identify students with research anxiety. Moreover, this study contributes to the literature since there is no separate subject of study for the diagnosis of anxiety in our country. Suggested that in future studies increasing sample size and done on students other universities. Until be more representative the demographics specifications sample of limitations this study Self-reporting of participants and reliance of the responses to the feelings of people at the moment of accountability.

\section{Acknowledgments}

This research was extracted from the MSc. thesis of the first author, in the Department of Educational, Faculty of Education and Psychology, Ferdowsi University of Mashhad, Mashhad, Iran.

\section{Conflict of Interest}

The authors declared no conflicts of interest 


\title{
ساخت و اعتباريابى مقياس اضطراب از ئوهش براى دانشجويان
}

\author{
فاطمه غلامى بورنك'، "بهروز مهرامّ، حسين كارشكى"

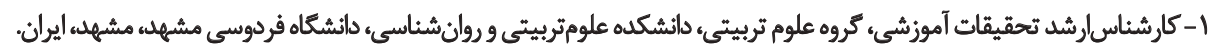

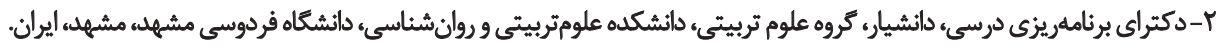

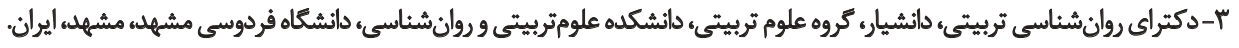

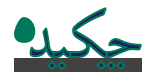

هداق هدف از اين مطالعه، ساخت و اعتباريابى مقياس اضطراب الز بروهش براي دانشجويان دانشكاه فردوسى مشهد بود.

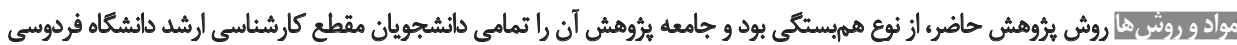

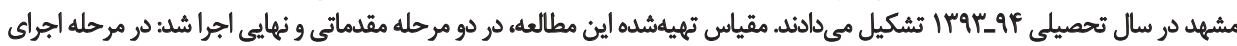

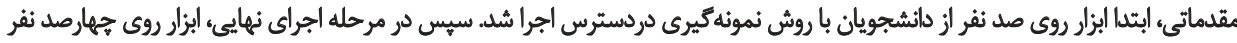

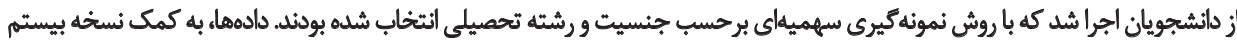

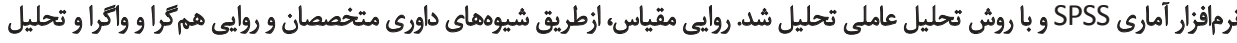

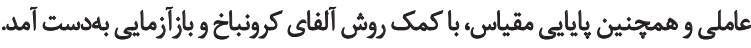

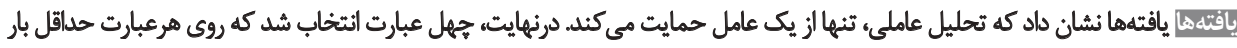

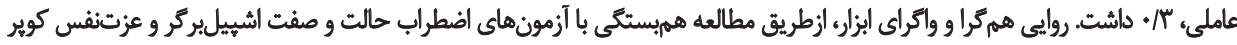

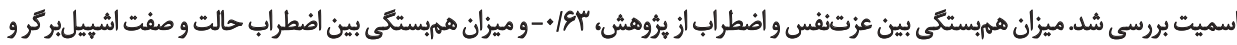

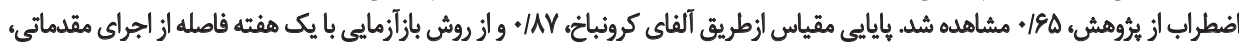

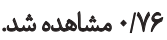

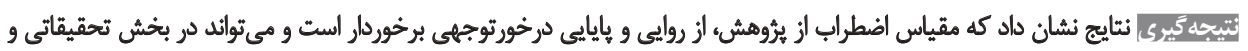

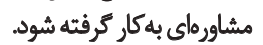

تاريخ دريافت: وF شهريور

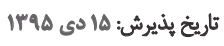

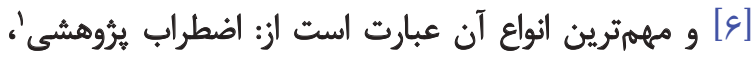

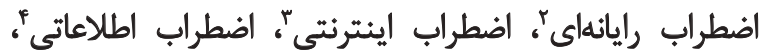

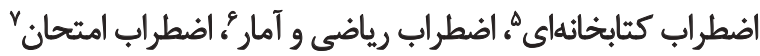

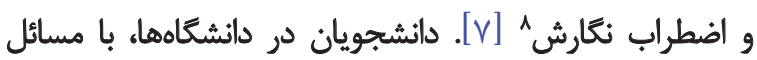

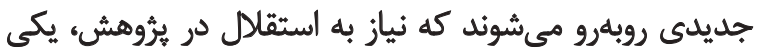

\footnotetext{
1. Research anxiety

2. Computer anxiety

3. Internet anxiety

4. Information anxiety

5. Library anxiety

6. Math anxiety \& statistics anxiety

7. Test anxiety

8. Writing anxiety
}

dateo

اضطراب به حالتى در فرد اشاره مى كند كه باموجب آن، وى دي

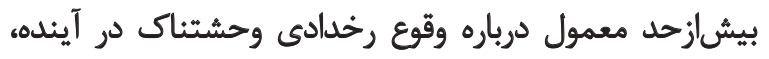

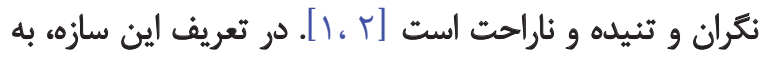

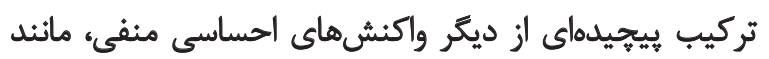

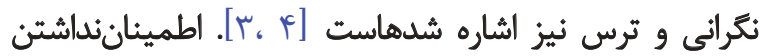

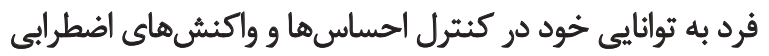

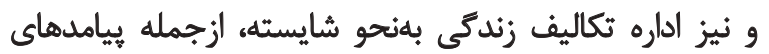

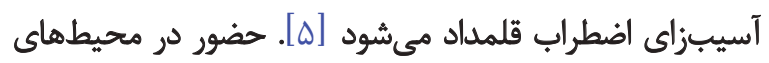

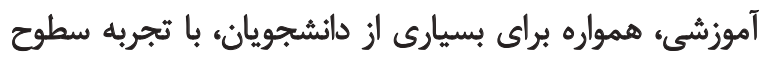

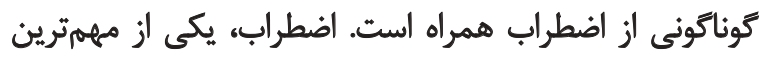

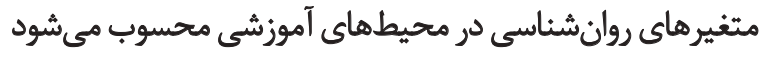




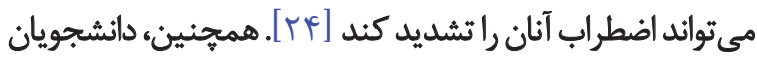

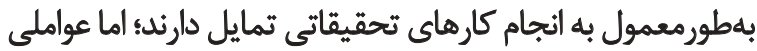

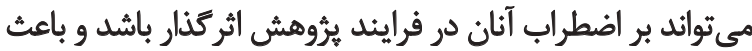

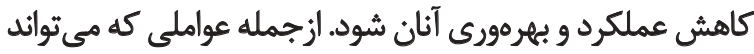

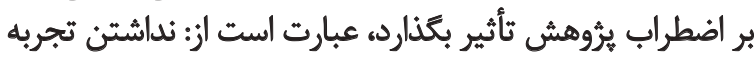

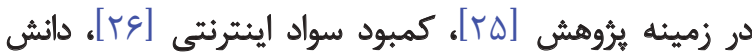

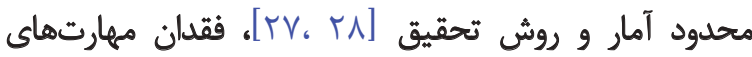

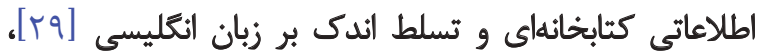

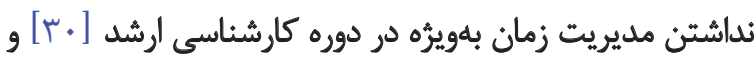

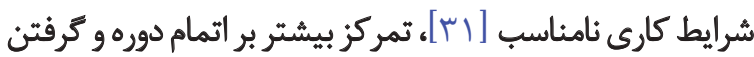

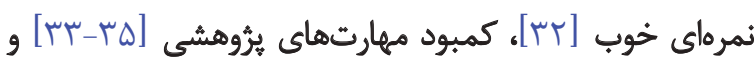

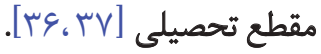

در داخل و خارج كشور، اضطراب از ئرهش موضوع برخى

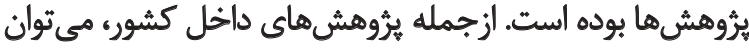

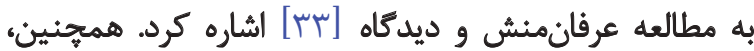

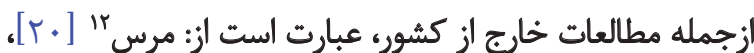

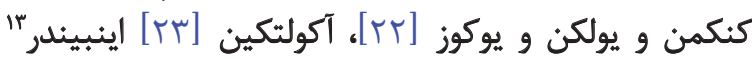

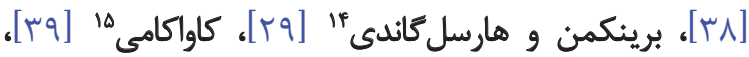

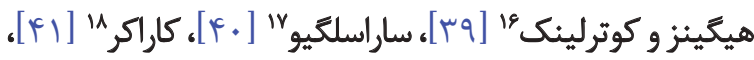

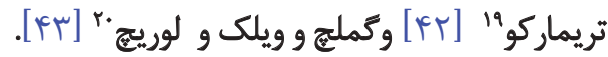

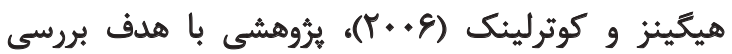

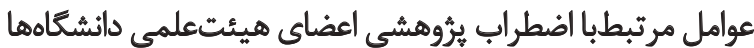

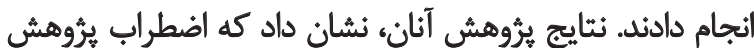

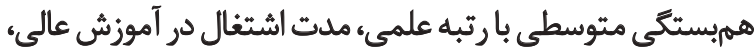

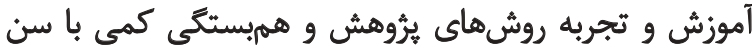

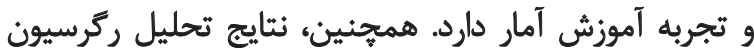

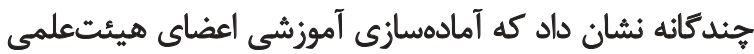

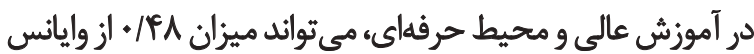

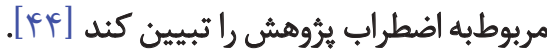

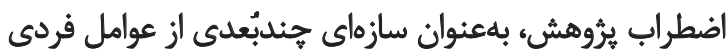

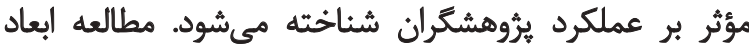

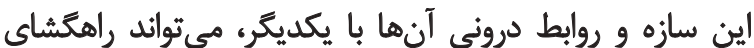

\section{Merç}

13. Einbinder

14. Brinkman \& Hartsell-Gundy

15. Kawakami

16. Higgins \& Kotrlik

17. Saracaloğlu

18. Kracker

19. Trimarco

20. Gmelch, Wilke, \& Lovrich
ز آنهاست. احساس اطمينان دانشجو دربرابر توانايي و تصور از براز

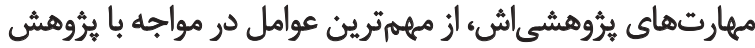

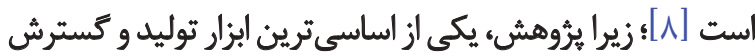

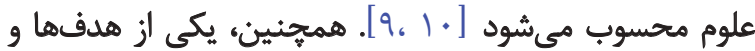

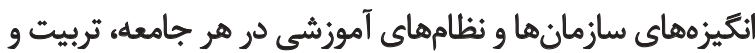

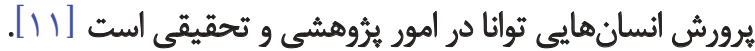

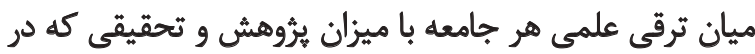

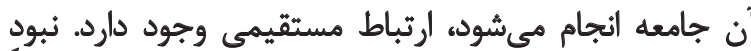

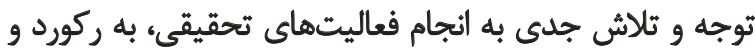

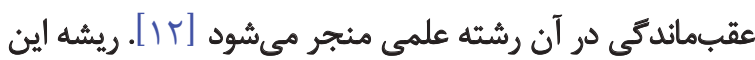

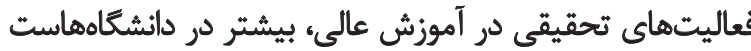

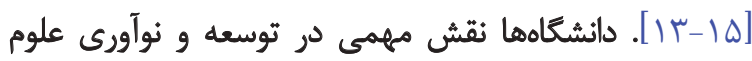

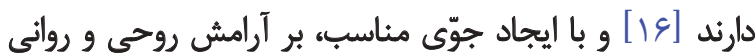

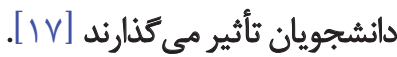

مطالعات نشان مى دهد كه بروهش با موانع بازدارندهاى مواجه

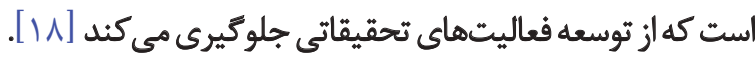

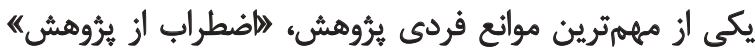

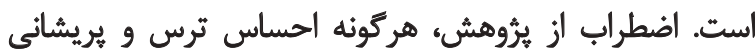

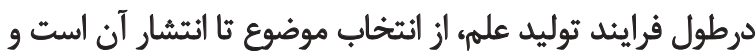
حتى بازخوردهايي رادربرمى كيرد كه يس يساز انجام مطالعه، أزسوى

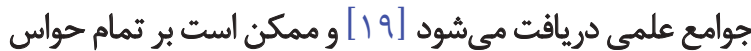

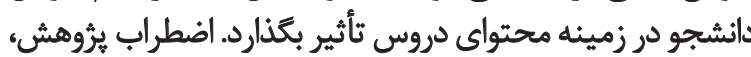

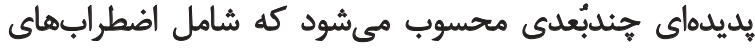

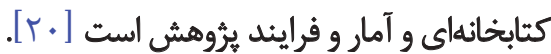
بولين، لى، كلنماي و يون" اضطراب يُروهش رابخشى از نكرش

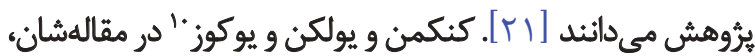

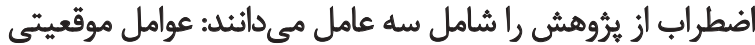

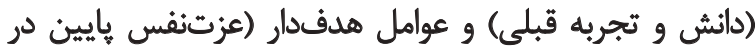

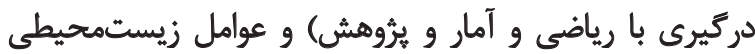

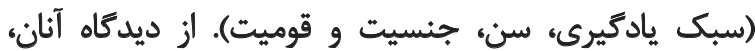

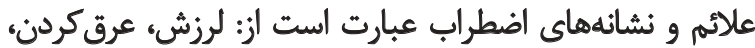

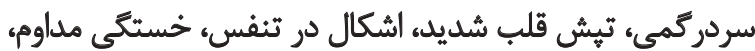

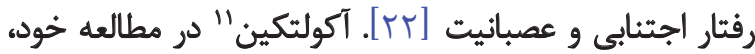

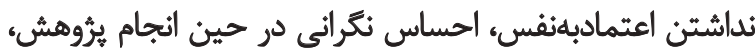

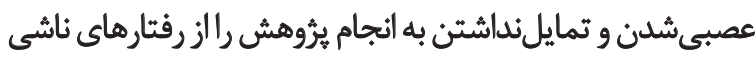

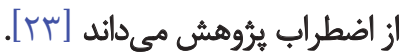

باوجوداين، تحقيقات نشان داده كه درحدود نيمى از افراده

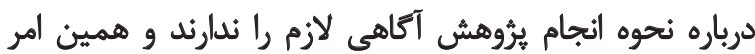

9. Bolin, Lee, GlenMaye, \& Yoon 10. Konokman, Yelken, \& Yokuş 11. Akcoltekin 


\section{$\mathrm{n}=\frac{1874 \times 3 / 30 \times 97}{1874 \times 1 / 45^{2}+3 / 30^{2} \times 97}$}

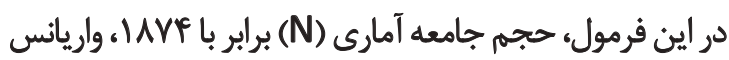

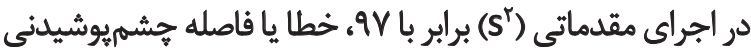

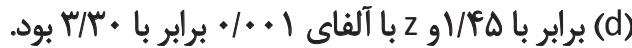

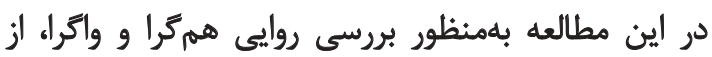

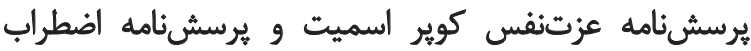
اشيّيل بركر استفاده شيد.

\section{يوسش نامه عزتثنفس كوير اسميث}

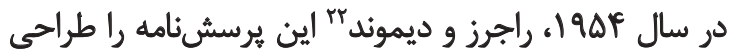

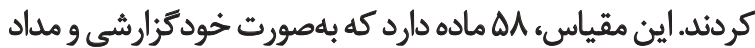

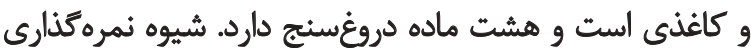

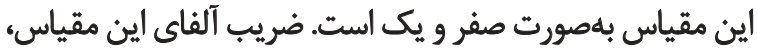

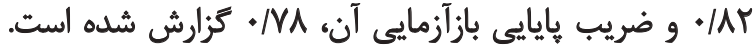

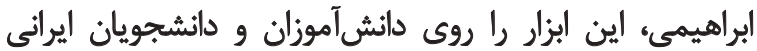

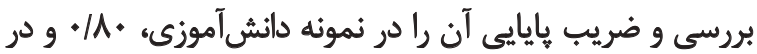

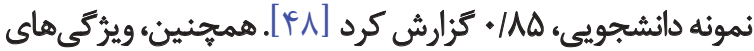

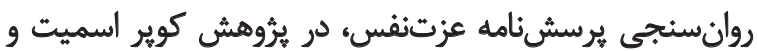

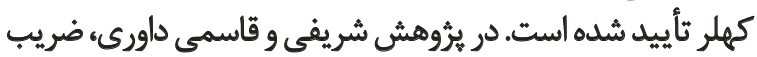

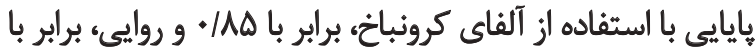

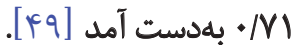

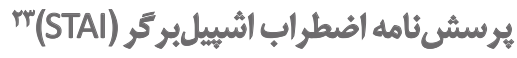

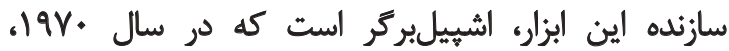

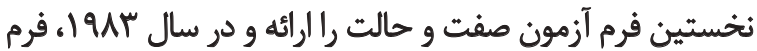

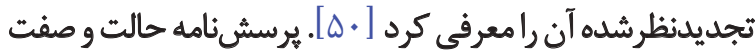

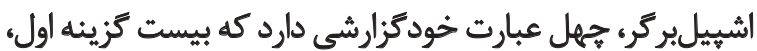

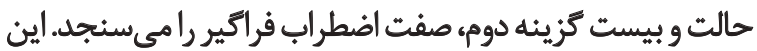

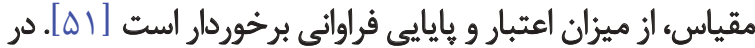

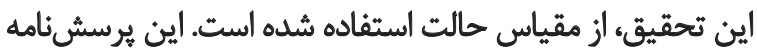

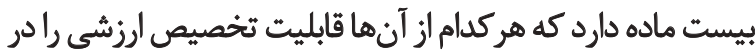

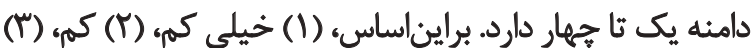

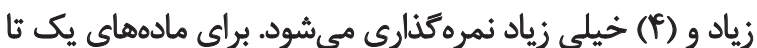

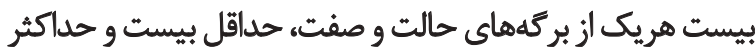

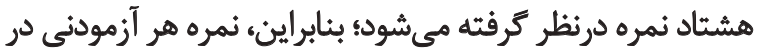

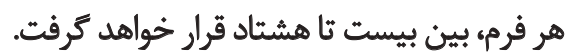

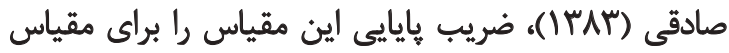

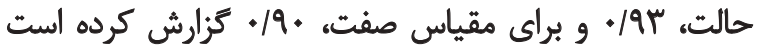

21. Cooper Smith Self Esteem Inventory

22. Rojers \& Dymund

23. Spielberger State-Trait Anxiety Inventory
يُروهش هايى ديكر و كسترش دانش سازمانيافته در اين حيطه

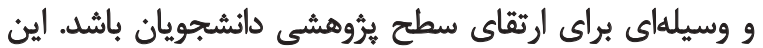

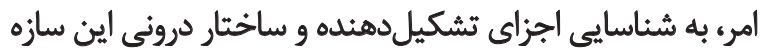

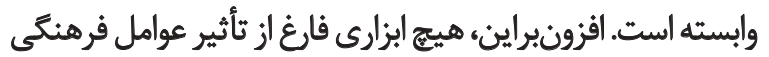

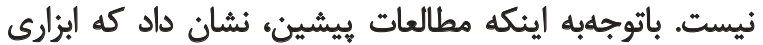

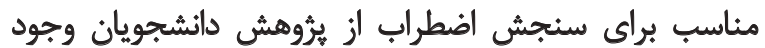

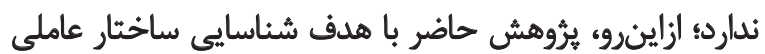

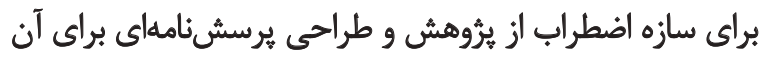

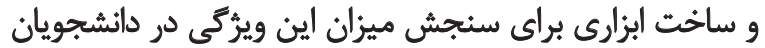

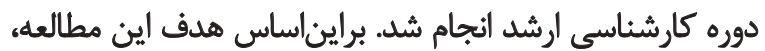

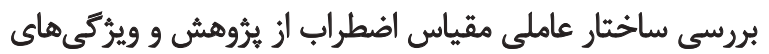
روانسنجى آن بود.

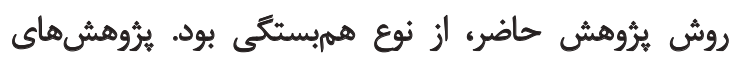

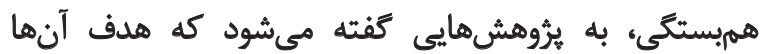

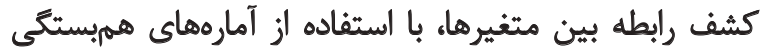

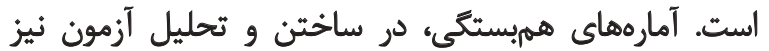

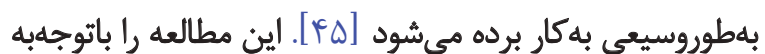

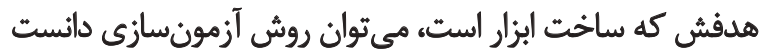

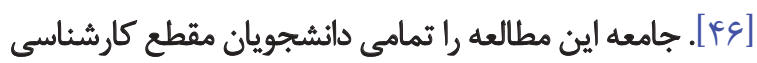

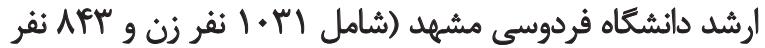

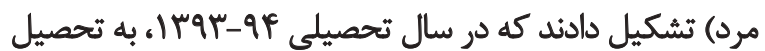

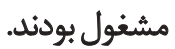
در مطالعه حاضر، معيار ورود آزمودنى ها عبارت بود از: تحصيل

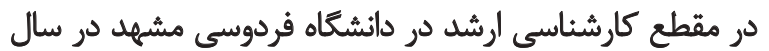

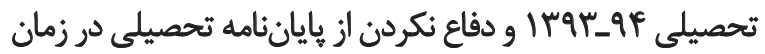

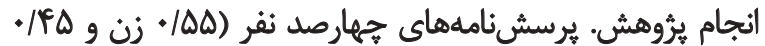

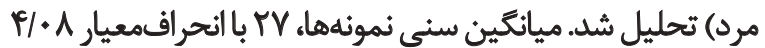

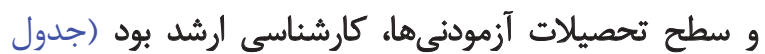

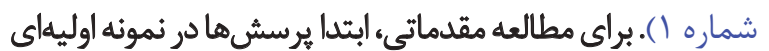

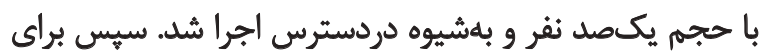

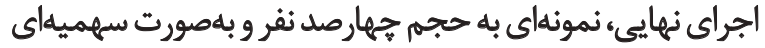

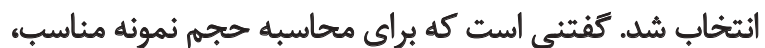

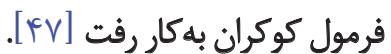

در اين يرؤهش، براي تجزيهوتحليل دادهاي جمعآورىشدانه،

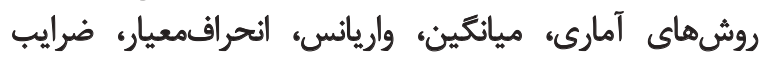

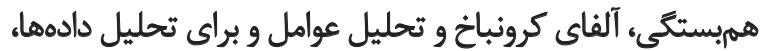
نسخه بيستم نرمافزار آمارى SPSS به كار رفت $\mathrm{n}=\frac{\mathrm{Nt}^{2} \mathrm{~s}^{2}}{\mathrm{Nd}^{2}+\mathrm{t}^{2} \mathrm{~s}^{2}}$ 


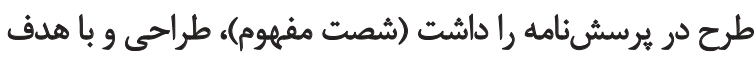

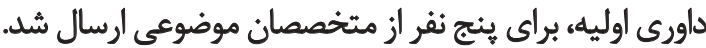
r. در اين مرحله، طراحى گويههاي برسشائنامه براساس

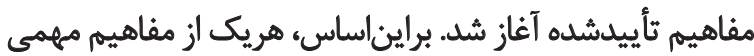

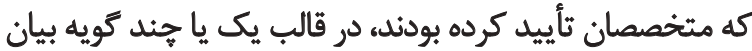

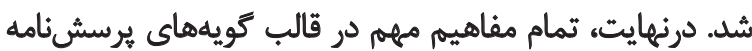

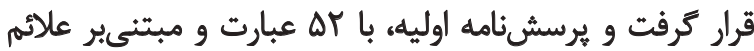

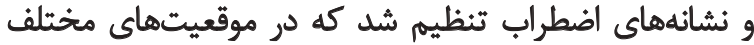

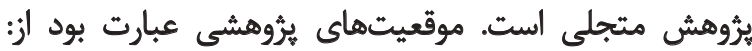

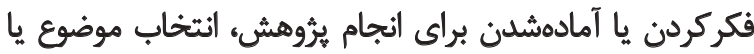

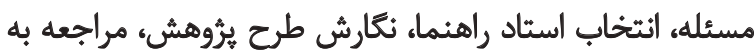

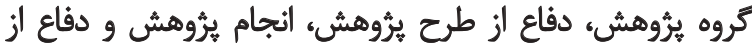

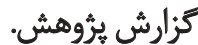

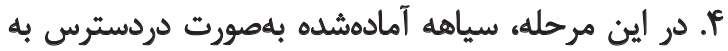

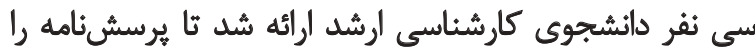

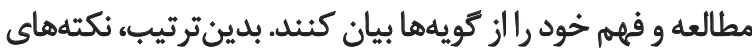

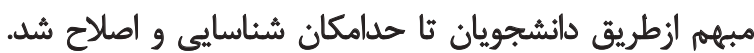

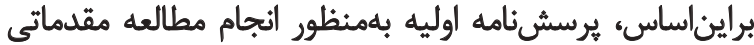

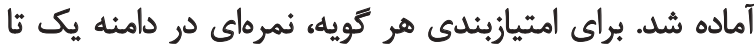

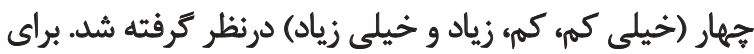

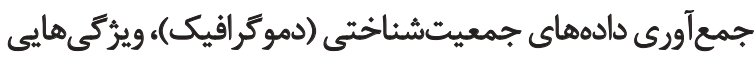

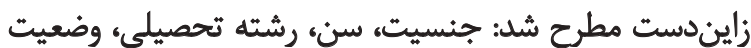

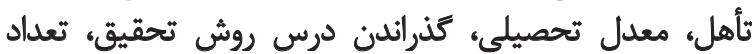

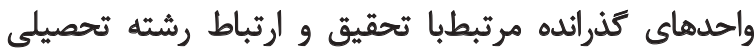
كارشناسى با تحصيل در مقطع كارشناسى ارشد، وضعيت اشتئ اشتغال

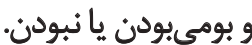

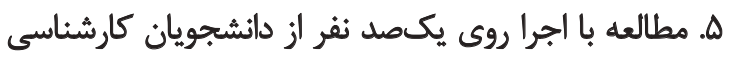

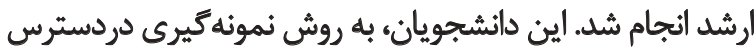

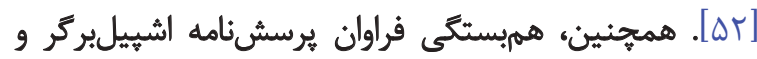

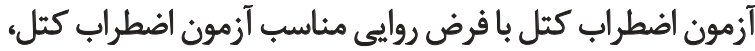

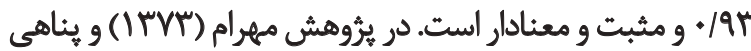

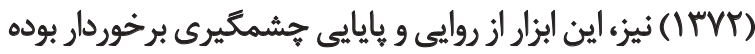

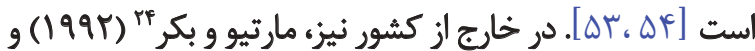

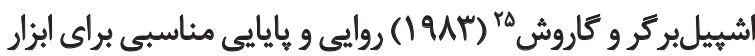

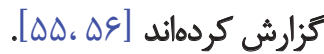
براى ساخت و سنجش روايى و پايايى يرسشنامه اضطراب از يزؤهش، مراحل زير انجام شد:

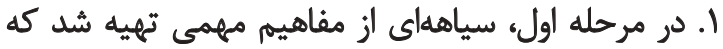

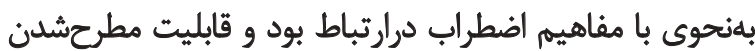

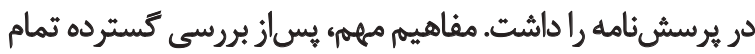

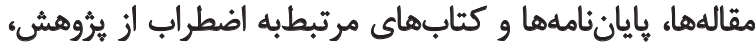

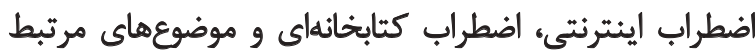

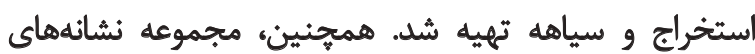

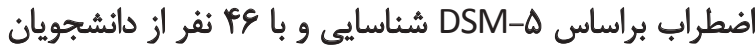

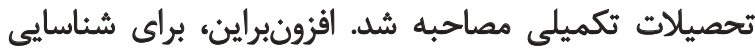

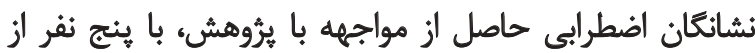

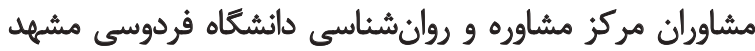

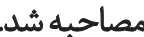

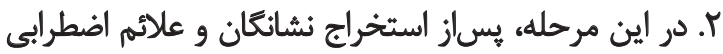

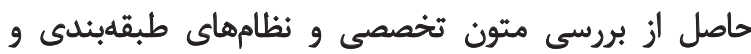

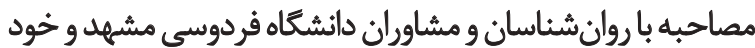

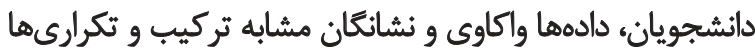

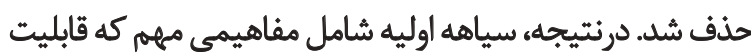

24. Marteau \& Bekker

25. Spielberger \& Gorsuch

جدول ا. نمونه بررسى شده برحسب جنسيت و رشته تحصيلى در دائشويان دانشكاه فردوسى مشهد

\begin{tabular}{|c|c|c|c|c|}
\hline \multicolumn{2}{|c|}{ جنسيت } & \multirow{2}{*}{ تعداد نمونه } & \multirow{2}{*}{ هاتشكده } & \multirow{2}{*}{ رديف } \\
\hline 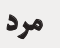 & ز & & & \\
\hline 1. & if & mp & الهيات و معارف اسلامي & 1 \\
\hline r. & err & rer & ادييات و علوم|نسانيى & r \\
\hline$M$ & re & pr & علومادارى و اقتصادى & r \\
\hline$\checkmark$ & r & rq & علومتربيتى و روانشناسيى & f \\
\hline ra & $p q$ & $w$ & 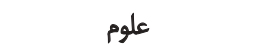 & 8 \\
\hline v. & $r$ & qv & مهنسى & $\wedge$ \\
\hline ro & rA & er & كشاوزي & 1. \\
\hline
\end{tabular}

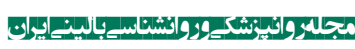




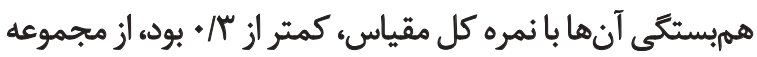

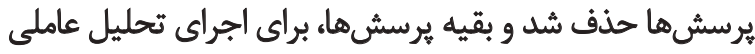

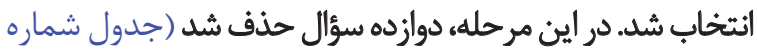

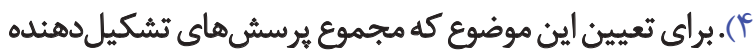

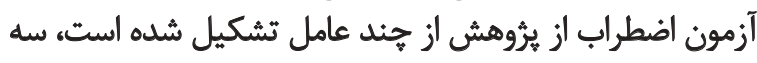

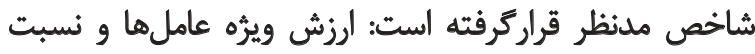

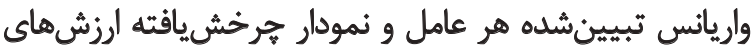

$$
\text { ويزه (اسكرى) " (جدول شماره س). }
$$

يافتهها

نتايج نشان داد كه ميتوان يازده عامل را باتوجهبه تعداد

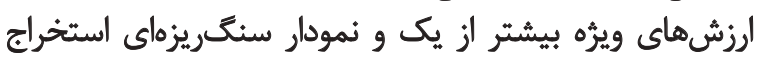
كرد (تصوير شماره (). براى تفكيك و استخراج بهتر عوامل،

26. Scree
انتخاب شده بودند. هدف از انجام اين مطالعه، شئاسايي كويههاي

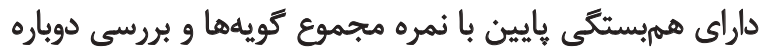

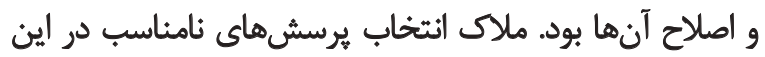

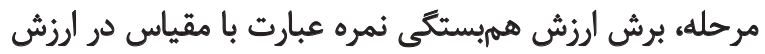

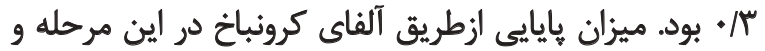

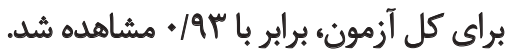

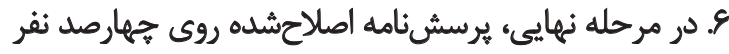

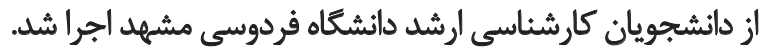

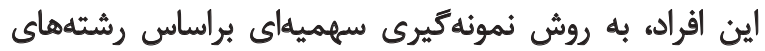
تحصيلى و جنسيت انتخاب شده بودند (جدول شماره ()). بهمنظور تحليل دادهها و انجام تحليل عاملى، مناسببودن

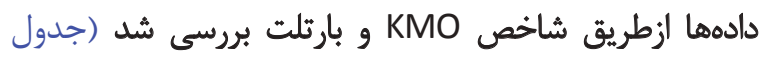

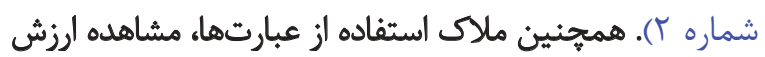

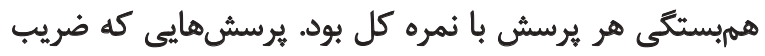

جدول Y. شاخص KMO و آزمون كرويتبارتلت براى مقياس اضطراب از ثيروهش

\begin{tabular}{|c|c|c|c|}
\hline \multicolumn{4}{|c|}{ آزمون بارتثلت } \\
\hline أزمون KMO & سطح معنى دارى & درجه آزادى & كاى دو \\
\hline$\cdot / M$ & $+1+\infty 1$ & $V_{1}$. & retVIg \\
\hline
\end{tabular}

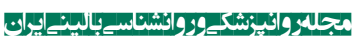

جدول ×. بارهاى عاملى برسش هاى مقياس اضطراب از ئروهش

\begin{tabular}{|c|c|c|}
\hline 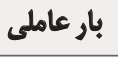 & محتواى كويه & شماره كويه \\
\hline.$/ 4 T$ & هر كاه فكر مى كنم كه بايل طرحى ثلدوين كنه، عصبى و آشفته مى شوم. & 1 \\
\hline . Met & با الديشيدن به كارهايى كه بايد براي أججام يروهشم أنجام دهمه، بى خوابي بلهسراغم هي آيد. & $r$ \\
\hline.$/ 19 q$ & هنكامى كه به جمع آورى مطالب يرؤوهم فكر مى كنم، دجار اغتشاش ذهنى مي شوم. & $r$ \\
\hline.$/ \Delta 9$ & درباره ناتوانى از انجامدادن يُوهش، زياد فكر مى كنهم. & p \\
\hline.$/ \mathrm{MA}$ & هركاه به انجام كارى بئوهشى فكر مي كنم، احساس اميدوارى مى كنم. & $\Delta$ \\
\hline • & در انتخاب موضوع مناسب، هيج نكرائى و دلمرهاي ندارم. & 8 \\
\hline$\cdot / \% \Delta$ & از فكركردن مداوم به انتخاب موضوع، دجار سردرد مى شوم. & $\gamma$ \\
\hline$+/ M$ & جنانجه متوجه شوم كه موضوعم تكرارى است، بيشازحد معمول نكران خواهم شد. & $\Lambda$ \\
\hline$\cdot / \pi$ & از اينكه بايد زياد بهل استاد راهنما مراجعه كنم، عصبى مى شوم. & 9 \\
\hline$\cdot / \mathrm{kg}$ & از ايثكه نتوانم طرح (يرويوزال) را در زمان مثاسب تحويل دهم، دلهره دارم. & 1. \\
\hline . $/$ er & 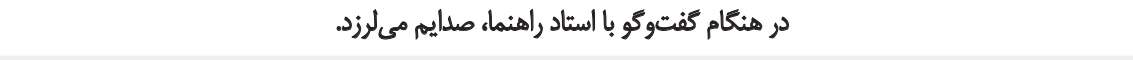 & 11 \\
\hline$\cdot / M^{\prime \prime}$ & سعى هي كثم از موقعيتهايع دورى كزينم كه مرا به انجام مرثوهش وادار هي كند. & $\pi$ \\
\hline $.17 \%$ & فكر هي كنم در هنكام جستوجو براى ييشينه نظرى و تحقيقات أنجامشده قبلى، احساس آشفتكى به من دست خواهد داد. & ir \\
\hline$+/ 4 A$ & از اينكه جه كسانى داوران يايان نامهام شونده احساس دلمره مى كنم. & if \\
\hline
\end{tabular}




\begin{tabular}{|c|c|c|}
\hline 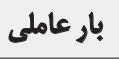 & محتواى تويه & شماره كَيه \\
\hline - /AF & فكر هي كنم از شدت نكراتى درباره دفاع از طرحمه دهار كرفتكى عضلانى شوم. & 10 \\
\hline .48 & حتى هنكامي كه به موضوعم هسلط هستمى احساس دلمهره و دل شوره خواهم داثت. & \&) \\
\hline.$/ 4 A$ & 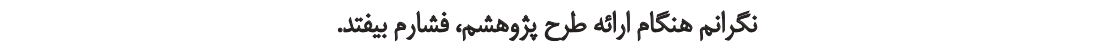 & iv \\
\hline.$|\Delta|$ & فكر مى كنم وقتى بخواهم از طح بئوهشم دفاع كنم، دهار بي خوابى مى شوم. & M \\
\hline.$/ M$ & 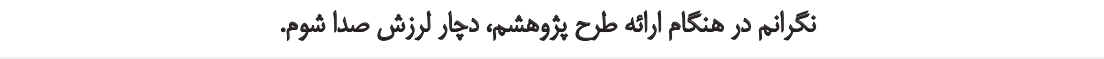 & 19 \\
\hline.$/ 4 \cdot$ & هنكامى كه كار يثوهشىام را انجام مى دهم، احساس ثاثوائى مى كنم. & r. \\
\hline.$/ \mathrm{ke}$ & هركاه تصميم هى كيرم كه يروهشى را انجام دهم، احساس مى كتم كه از جمع دوستان و اطرافيان فاصله كرفتهام. & $M$ \\
\hline$+/ 4$ & 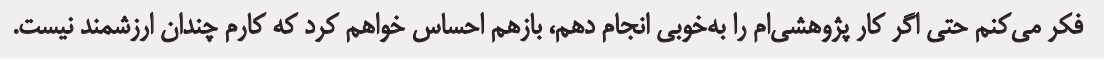 & ri \\
\hline$\cdot / \mathrm{HV}$ & جنانجهه نمونهام جواب مدنظر را ندهد، عصبى خواهم شد. & 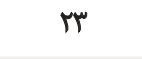 \\
\hline.$/ 16$ & ترعم از اين است كه نكرائى بيشازحد قبل از شروع جلسه دفاع، مرادهار دل بههمخوردكى كند. & re \\
\hline.$/ \% \Delta$ & اميلوار ثيستم كه در نكارش كزارش برؤهشىام، موفق باشمه. & TA \\
\hline.$/ 81$ & نكرائم قبل از شروع جلسه دفاع، احساس بى قرارى به من دست دهذ. & re \\
\hline$\cdot / \Delta V$ & اطمينان دارم در هنكام نوشتن ييشينه، دهار بي خوابى مىشوم. & rV \\
\hline.$/ \mathrm{QV}$ & فكر مى كنم باوجود تلاش فراوان، نخحواهم توائست هنكام ارائه كزارش يزوهشهم، تمركز حواس داشته باشهم. & rA \\
\hline - /af & مى دانم از فكر اينكه نمرهاي كم در جلسه دفاع بكيرم، عصبي خواهم شل. & rq \\
\hline.$|8|$ & فكر مى كثم در جلسه دفاع براى ياسخدادن به هبرسش هاي داوران، احساس ناتواثى به من دست خواهد داد. & r. \\
\hline .180 & 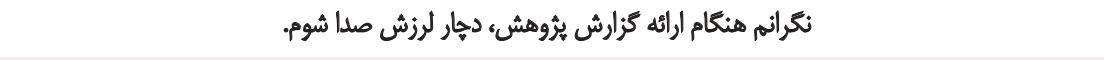 & 省 \\
\hline - laf & كمان مى كنم هنكام دفاع، دجار تيش شديد قلب خواهم شد. & m \\
\hline.$/ \Delta$ & درباره شكست در جلسه دفاع زياد فكر مي كثم. & $M$ \\
\hline .181 & فكر مي كنم در هنكام ارائه كزارش برو هشمه جهرهام سخ شود. & $m$ \\
\hline .181 & 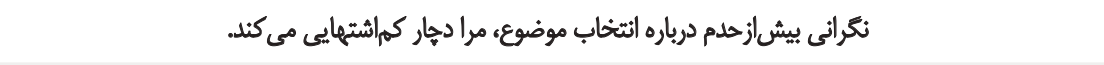 & ra \\
\hline.$/ 4$ & اطمينان دارم بهخوبى از كزار ش يُوهشهء دفاع خواهم كرد. & re \\
\hline$* / M$ & اميدوارم بتوائم طرحم را در زمان تعيين شده، دفاع كنم. & W \\
\hline$\cdot / M$ & 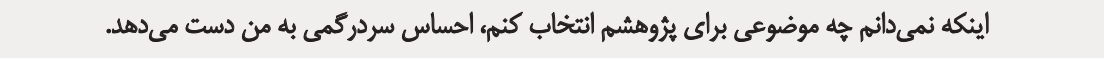 & ra \\
\hline$\cdot / A F$ & از فكر كردن درباره طرح يُوهشىام، دجار سردرد مي شوم. & rq \\
\hline.$/ 19$ & با خودم فكر هي كنم نكرانى زياد در جلسه دفاع از باياننامه، هرا دجار حالت تهوع خواهد كرد. & f. \\
\hline
\end{tabular}

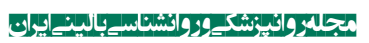

بارهاى مربوط، تمام برسش هاى برسشنامه در قالب يك عامل

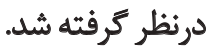

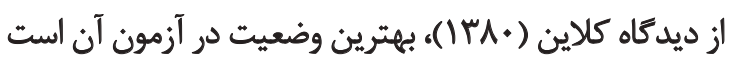

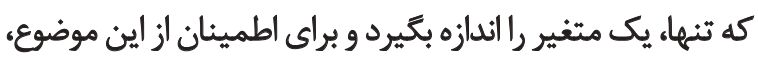

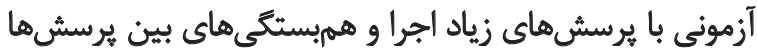

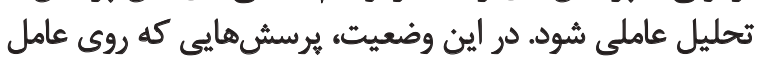

از جرخش واريماكس استفاده شد؛ اما باتوجهبه قدرت تبيين

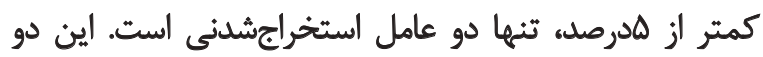

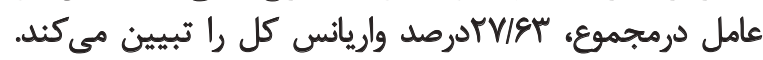

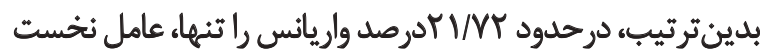

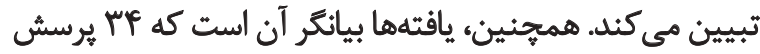

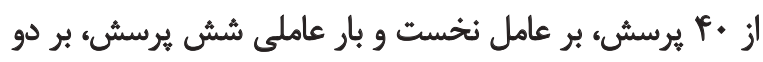

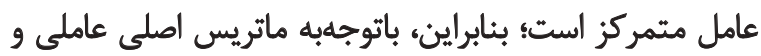




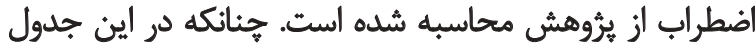

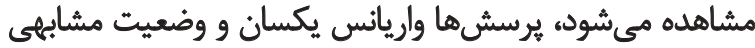

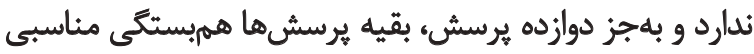

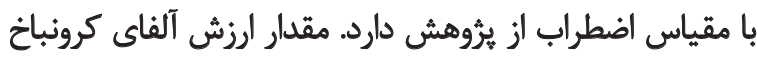

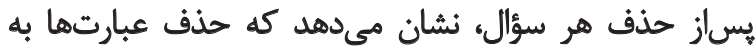

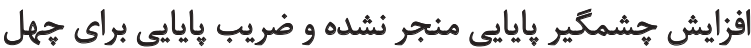

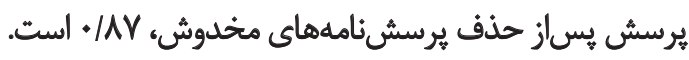
نمره خامى راكه يساز اجراى آزمون بهدست مى آيد، نمى توان

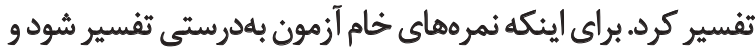

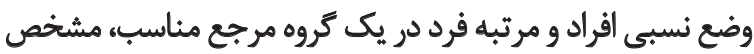

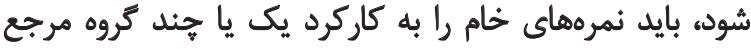

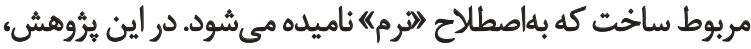

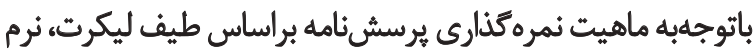

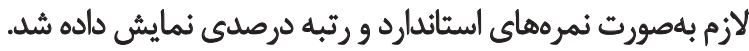

بحث

اضطراب، حالتي است كه فرد بيشازاندازه درباره وقوع رخدادى

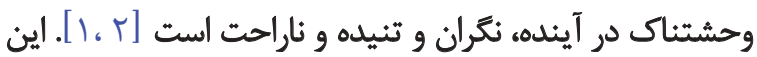

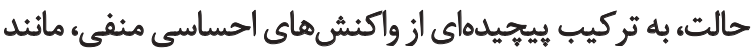

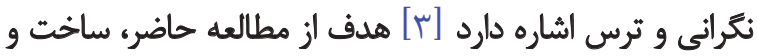

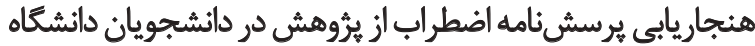

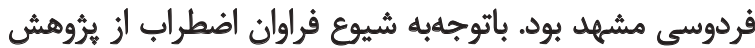

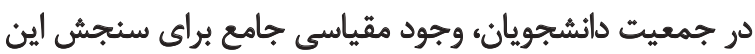

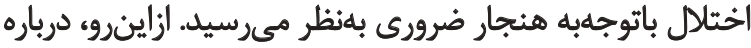

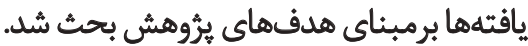

هدف اول يثوهش، بررسى ساختار عاملى مقياس اضطراب از

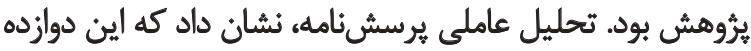

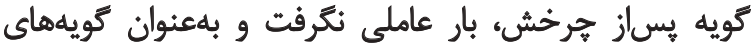

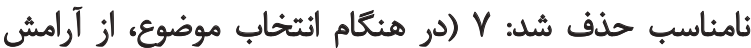

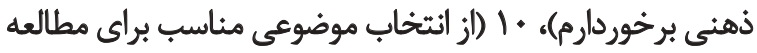

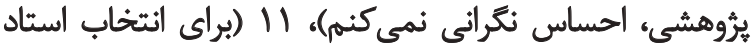

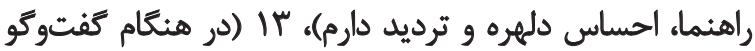
با استاد راهنما، احساس راحتى مئى كنيم)،

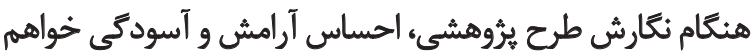

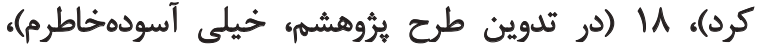

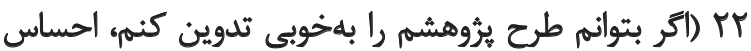

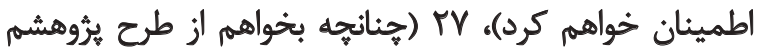

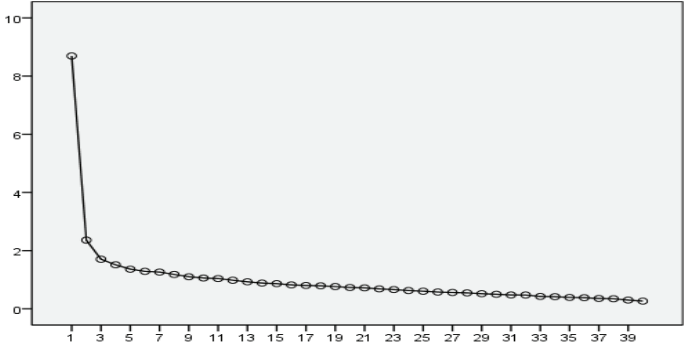

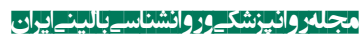

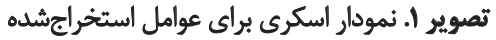

كلى بار داشته باشد، براى آزمون انتخاب مىشود. همان طور كه در

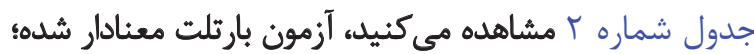
بنابراينء امكان انجام تحليل عاملى وجود دارد.

جدول شماره با، نشاندهنده جهل عبارتى است كه بعد از

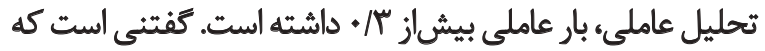

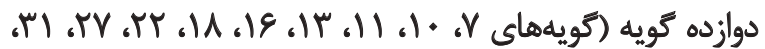

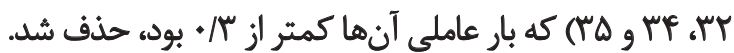
براى بررسى روايى آزمون، از تحليل عاملى و داورى متخصصان

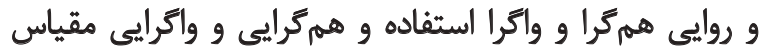

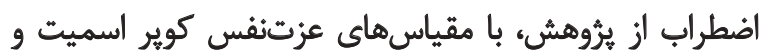

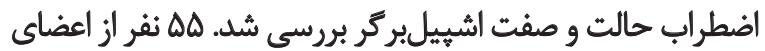

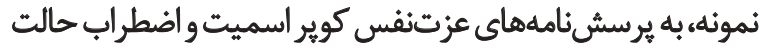

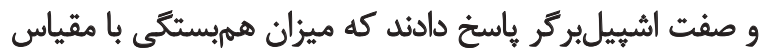

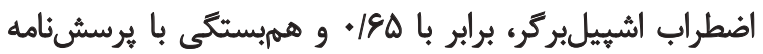

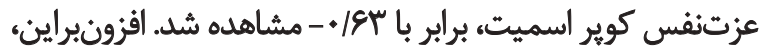

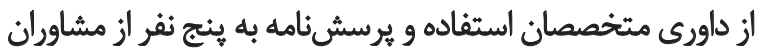

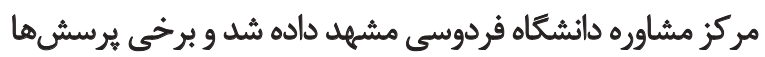
كه از كويايى لازم برخوردار نبود، اصلاح شد مدان

براي مطالعه باياييى، از همسانى درونى و ثبات استفاده شد.

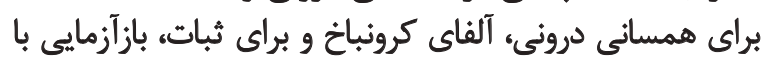

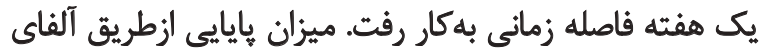

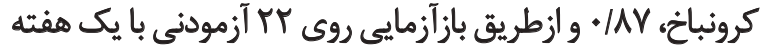

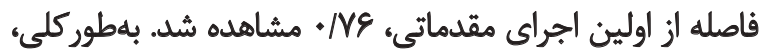

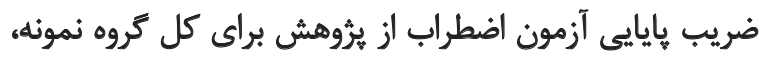

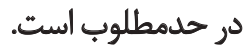

در جدول شماره أ، ضريب آلفاى كرونباخ براى كل مقياس

جدول F. ضريب آلفاى كرونباخُ براي كل مقياس اضطراب از يُرُوهش

ضريب آلفاي كرونباخ

$\cdot / 9 r$
تعداد كَّيه

r.

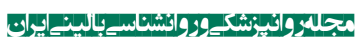


رتبه درصدى نمره Z و t قابليت تفسير نمرههاى آزمون اضطراب

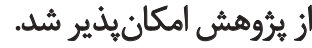

تتيجليَّيرى

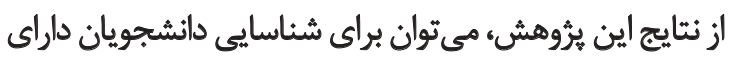
اضطراب از يثروهش استفاده كرد. اين مسئله، باتوجهبه ائنائ اينكه

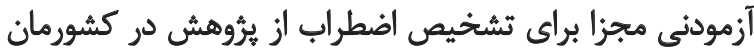

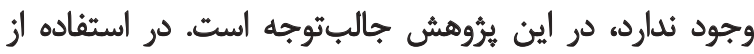

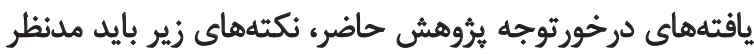

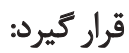

از در تهيه جدولهاى هنجارى، معرفبودن و حجم نمونئه

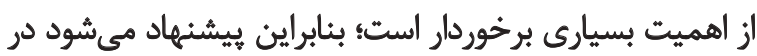

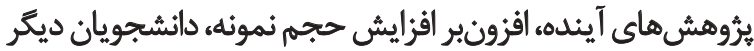

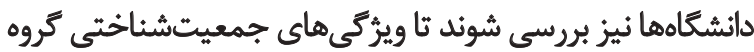

$$
\text { نمونه معرفتر شودي؛ }
$$

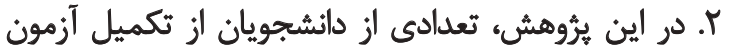

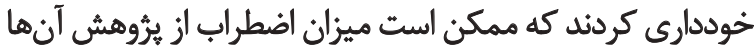

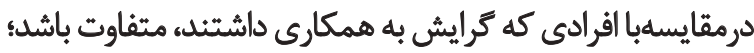

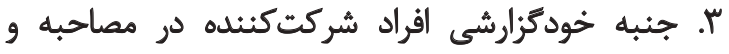

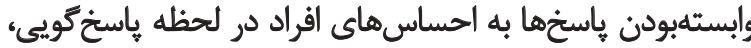

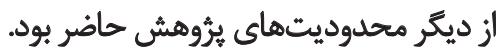

$$
\text { سياسكّارى }
$$

اين مقاله بركرفته از هايان إنامه مقطع كارشناسى ارشد نويسنده

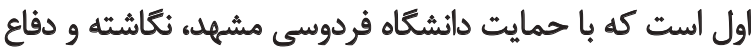

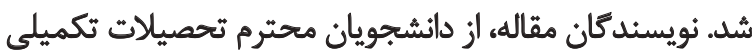

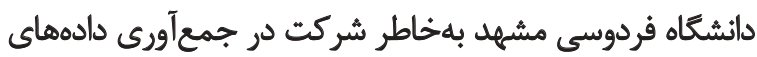

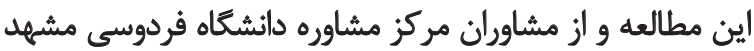

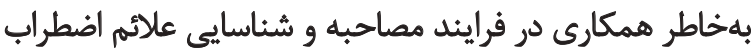

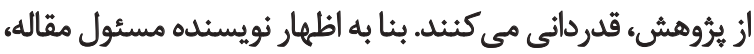
تعارض منافع وجود نداشته است
دفاع كنمه، احساس آرامش خواهم كرد)، اب (درباره تحليل كار

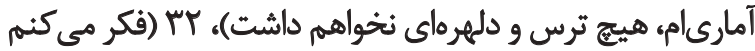

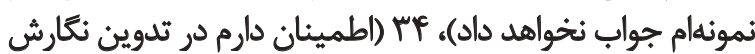

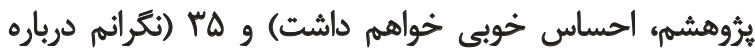
تحليل كار آمارىام، دجار بـى خوابى شوم).

هدف دوم يثروهش، بررسى ويزّكى هائى روانسنجى (روايى و و

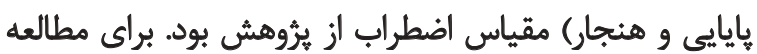

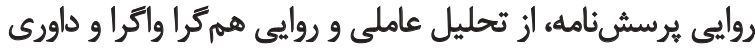

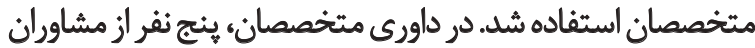

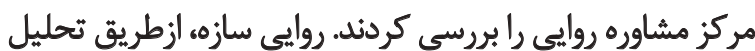

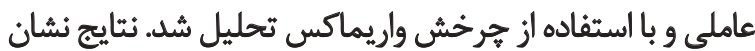

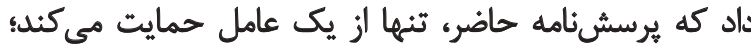

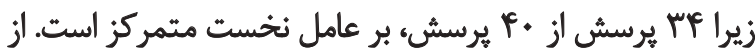

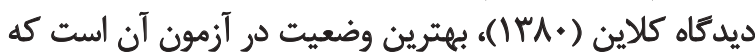

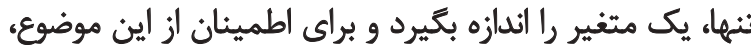

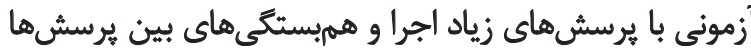

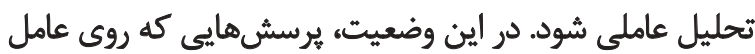

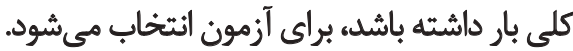

درباره روايى همثرا و واكرا، همبستكى با مقياس اضطراب

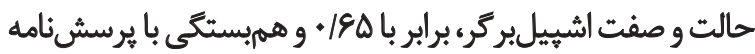

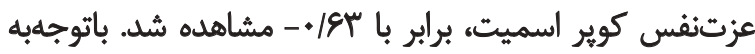

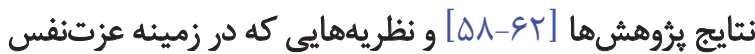

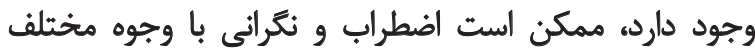

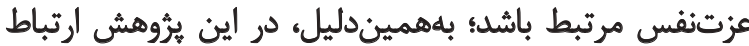

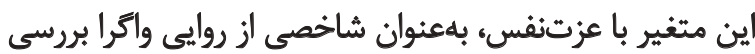

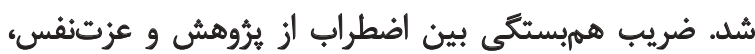

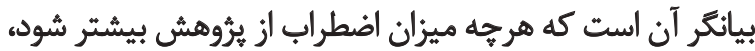

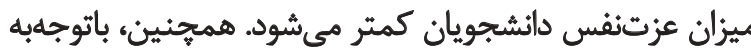

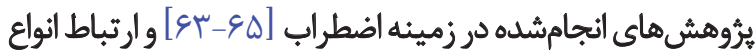

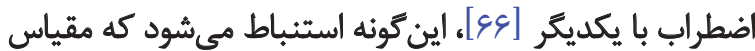

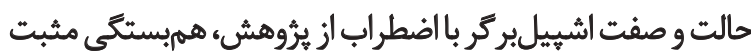

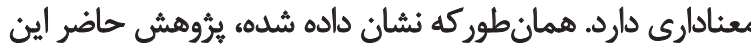

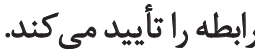

هاياييى اين مقياس، ازطريق ضريب همسانى درونى يرسشها

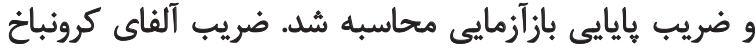

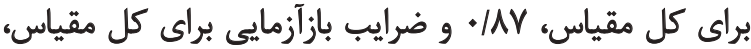

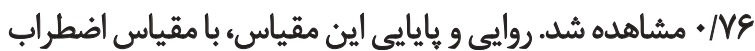

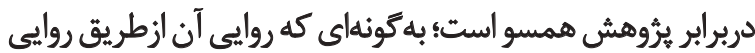

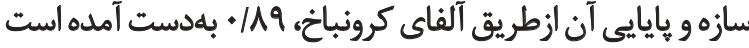

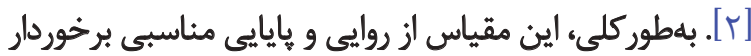

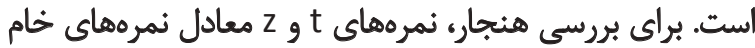

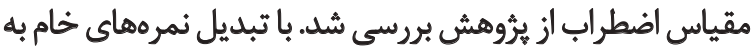


بيوست ا. يرسشنامه اضطراب از يثروهش

\begin{tabular}{|c|c|c|c|c|c|}
\hline خيلى زياد & 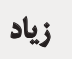 & S & خيلى كم & كويه & ش تشماره \\
\hline & & & & هركاه فكر مي كنم كه بايد طرحى تلوين كنم، عصبى و آشفته مى شوم. & 1 \\
\hline & & & & با انديشيدن به كارهايع كه بايد براى أجام يثروهشم أنجام دهم، بيى خوائي بلهراغم مي آيلد. & r \\
\hline & & & & هنكامى كه به جمع آورى مطالب برؤهشم فكر مي كثم، دهار اغتشاش ذهنى هي شوم. & $r$ \\
\hline & & & & درباره ناتواني از انجامدادن بروهش، زياد فكر هي كنم. & f \\
\hline & & & & هر كاه به انجام كارى يُروهشى فكر هي كنم، احساس اميدوارى هي كنه. & $\Delta$ \\
\hline & & & & در انتخاب موضوع مناسب، هيج نكراثي و دليهراي ندارم. & 8 \\
\hline & & & & از فكركردن مداوم به انتخاب موضوع، دهار سردرد مىشوم. & $\gamma$ \\
\hline & & & & جنانجه متوجه شوم كه موضوعم تكرارى است، بيشازخد معمول نكران خواهم شد. & $\wedge$ \\
\hline & & & & از اينكه بايد زياد به استاد راهنما مراجعه كنه، عصبى مى شوم. & 9 \\
\hline & & & & از اينكه نتوائم طرح (يرويوزال) را در زمان مناسب تحويل دهمه دلهره دارم. & 1. \\
\hline & & & & در هنكام كفتوكو با استاد راهنما، صدايمم مىلرزد. & 11 \\
\hline & & & & سعى مى كثم از موقعيتهايع دورى كزينم كه مرا به انجام يؤوهش وادار مى كثد. & ir \\
\hline & & & & 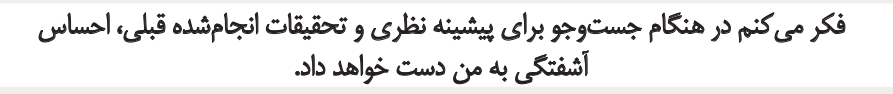 & Ir \\
\hline & & & & از اينكه جه كسانى داوران باياننامهام شوند، احساس دلمره مى كنم. & if \\
\hline & & & & فكر مى كثم از شدت نكرانى درباره دفاع از طرحم، دهار كرفتكى عضلانى شوم. & 10 \\
\hline & & & & حتى هنكامى كه به موضوعم مسلط هستمه احساس دلهره و دل شُوره خواهم داشت. & 18 \\
\hline & & & & نكراثم هنكام ارائه طرح يُروهشم، فشارم بيقتد. & iv \\
\hline & & & & فكر هي كنم وقتى بخواهم از طرح يُوهشم دفاع كنم، دهار بي خوابى هى شوم. & M \\
\hline & & & & نكرانهم در هنكام ارائه طرح هُوهشمه دهار لرزش صلا شوم. & 19 \\
\hline & & & & هنكامى كه كار يُؤوهشىام را انجام مى دهمه احساس ناتوائى مى كنه. & $r \cdot$ \\
\hline & & & & 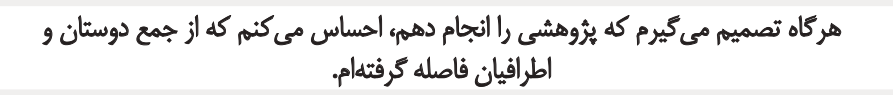 & $M$ \\
\hline & & & & فكر مى كنم حتى اكر كار بوروهشى ام را بهخوبى انجام دهمه بازهم احساس خواهم كرد كه كارم & MY \\
\hline & & & & 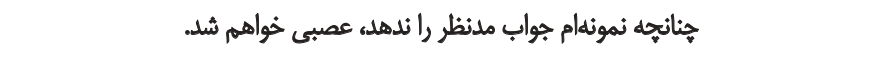 & ru \\
\hline & & & & ترسم از اين است كه نكرانى بيش ازخد قبل از شروع جلسه دفاع، مرا دجار دل بههمخوردكى & $M F$ \\
\hline & & & & اميدوار نيستم كه در نكارش كزارش يُروهشىامه موفق باشم. & ro \\
\hline & & & & نكرافم قبل از شروع جلسه دفاع، احساس بىقرارى به من دست دهلد. & re \\
\hline & & & & 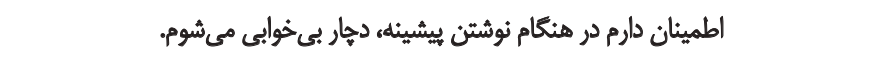 & M \\
\hline & & & & فكر مى كنم باوجود تلاش فراوان، نخواهم توانست هنكام ارائه كزارش يُورهشم، تمركز حواس & rA \\
\hline & & & & مى دانمم از فكر ايثكه نمرهاى كم در جلسه دفاع بكيره، عصبى خواهم شد. & rq \\
\hline & & & & فكر مي كثم در جلسه دفاع براي ياستخدادن به بردسش هائ داوران، الحساس ناتوائي به من & r. \\
\hline
\end{tabular}




\begin{tabular}{|c|c|c|c|c|c|}
\hline خيلى زياد & زياد & كمي & خيلى كم & تويه & شماره \\
\hline & & & & نكرانم هنكام الرائه كزّارش يُّوهش، دجار لرزش صدا شوم. & r \\
\hline & & & & كمان هي كنم هنكام دفاع، دهار تيش شديد قلب خواهم شُد. & m \\
\hline & & & & درباره شكست در جلسه دفاع زياد فكر مي كنم. & $m$ \\
\hline & & & & فكر مي كنم در هنكام ارائه كزارش يُّوهشمء جهرهام سخ شود. & me \\
\hline & & & & نكرائي بيشازحدم درياره انتخاب موضوع، مرادهار كم|شتتهايى مي كنلد. & ro \\
\hline & & & & اطمينان دارم بلهخوبى از كزارش يُروهشم، دفاع خواهمي كرد. & re \\
\hline & & & & اميدوارم بتوائم طرحم را در زمان تعيين شده، دفاع كنم. & r \\
\hline & & & & 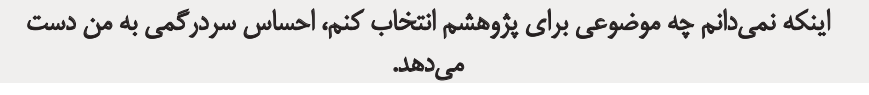 & ऍی \\
\hline & & & & از فكركردن درباره طح يُوهشى ام، دجار سردرد مي شوم. & rq \\
\hline & & & & با خودم فكر هي كثم نكرانى زياد در جلسه دفاع از باياينانامه، هرا دهار حالت تهوع خواهد كرد. & f. \\
\hline
\end{tabular}

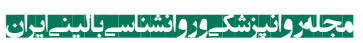




\section{References}

[1] Singewald N, Schmuckermair C, Whittle N, Holmes A, Ressler KJ. Pharmacology of cognitive enhancers for exposure-based therapy of fear, anxiety and trauma-related disorders. Pharmacology \& Therapeutics. 2015; 149:150-90. doi: 10.1016/j. pharmthera.2014.12.004.

[2] Yigit A. [The impact of computer assisted educational mathematics games on the basics and paternity in elementary level 2 in elementary education (Turkish)] [MSc. Thesis]. Adana: Çukurova University. 2007.

[3] Achim N, Kassim AA. Computer usage: The impact of computer anxiety and computer self-efficacy. Procedia - Social and Behavioral Sciences. 2015; 172:701-8. doi: 10.1016/j.sbspro.2015.01.422

[4] Yuksel M, Geban O. Examination of science and math course achievements of vocational high school students in the scope of self-efficacy and anxiety. Journal of Education and Training Studies. 2015; 4(1):88-100. doi: 10.11114/jets.v4i1.1090

[5] Halgin RP, Whitbourne SK. Abnormal Psychology: Clinical Perspectives on Psychological Disorders [Y. Seyed Mohammadi Persian trans]. Tehran: Ravan Publication; 2005.

[6] Headley C, Campbell M. Teachers' knowledge of anxiety and identification of excessive anxiety in children. Australian Journal of Teacher Education. 2013; 38(5). doi: 10.14221/ajte.2013v38n5.2

[7] Jiao QG, Onwuegbuzie AJ. The Relationship between Library Anxiety and Social Interdependence [Internet]. 2001 [2001 September 12]. Available from: https:/ / eric.ed.gov/?id=ED462079

[8] Lev EL, Kolassa J, Bakken LL. Faculty mentors' and students' perceptions of students' research self-efficacy. Nurse Education Today. 2010; 30(2):169-74. doi: 10.1016/j.nedt.2009.07.007

[9] Lauto G, Sengoku S. Perceived incentives to transdisciplinarity in a Japanese university research center. Futures. 2015; 65:136-49. doi: 10.1016/j.futures.2014.10.010

[10] Hutchins KK. Strengthening the development of communityuniversity partnerships in sustainability science research. Orono, Maine: University of Maine; 2013.

[11] Roomiyani Y, Mirshah Ja'fery E, Nasr Isfahani AR. [Organizational and educational obstacles pertaining to research activities of high-school teachers in the education department (Persian)]. Journal of Educational Psychology Studies. 2006; 3(4):59-74.

[12] Tabeyi S, Mahmoodian F. [Ethics in research (Persian)]. Ethics in Science and Technology. 2007; 2(1):49-54.

[13] Moyane SP. Research capacity needs of academic staff in the humanities at the University of Zululand [MSc. thesis]. Glenwood, Durban: University of Kwazulu-Natal; 2007.

[14] Gao S. The measurment of tertiary education quality in Indonesia through the education production model and policy recommendations for quality improvement [PhD thesis]. Pittsburgh, Pennsylvania: University of Pittsburgh; 2015.

[15] Kehm BM, Musselin C. The development of higher education research in Europe: 25 years of CHER. Dordrecht: Sense Publishers; 2013.

[16] Saral DG, Reyhanlioğlu D. An analysis of educational faculty students' research self-efficacy in terms of a number of variables. Procedia - Social and Behavioral Sciences. 2015; 174:1138-45. doi: 10.1016/j.sbspro.2015.01.729
[17] Spell CS, Arnold TJ. A multi-level analysis of organizational justice climate, structure, and employee mental health. Journal of Management. 2007; 33(5):724-51. doi: 10.1177/0149206307305560

[18] Hosseiny Shavoun A, Jahed H. [The viewpoint of faculty members on research obstacles at Tabriz University (Persian)]. Journal of Science \& Technology Policy. 2012; 4(4):49-64.

[19] Erfanmanesh M, Didegah F. [Researchers' and faculty members' research anxiety and its causes: Literature review (Persian)]. Journal of National Studies on Librarianship and Information. 2012; 23(1): 58-73

[20] Merç A. Research Anxiety among Turkish Graduate ELT Students. Current Issues in Education. 2016; 19(1).

[21] Bolin BL, Lee KH, GlenMaye LF, Yoon DP. Impact of research orientation on attitudes toward research of social work students. Journal of Social Work Education. 2012; 48(2):223-43. doi: 10.5175/ jswe.2012.200900120

[22] Konokman GY, Yelken T, Yokuş G. Preschool teacher candidates' research qualifications and anxiety level towards research Eurasian Journal of Educational Research. 2015; 15(60):57-74. doi: 10.14689/ejer.2015.60.4

[23] Alpturk A. High school students time management skills in relation to research anxiety. Educational Research and Reviews. 2015; 10(16):2241-9. doi: 10.5897/err2015.2345

[24] Basturk R, Karagul K, Karagul N, Dogan M. Predicting the academic acheivement of vocational college students. Journal of contemporary Education Academic. 2012; 1(1): 3-10.

[25] Harder J. Overcoming MSW students' reluctance to engage in research. Journal of Teaching in Social Work. 2010; 30(2):195-209. doi: $10.1080 / 08841231003705404$

[26] Stark RB, Cohen BE. Promoting positive student attitudes toward social work research using course web sites. Journal of Teaching in Social Work. 2007; 27(1-2):181-98. doi: 10.1300/ j067v27n01_12

[27] Pan W, Tang M. Students' perceptions on factors of statistics anxiety and instructional strategies. Journal of Instructional Psychology. 2005; 32(3):205-15

[28] Secret M, Ford J, Rompf EL. Undergraduate research courses: A closer look reveals complex social work student attitudes. Journal of Social Work Education. 2003; 39(3):411-22

[29] Brinkman SN, Hartsell-Gundy AA. Buildingtrust to relieve graduate student research anxiety. Public Services Quarterly. 2012; 8(1):26-39. doi: 10.1080/15228959.2011.591680

[30] Witt SL, Lovrich NP. Sources of stress among faculty: Gender differences. The Review of Higher Education. 1988; 11(3):269-84. doi: $10.1353 /$ rhe.1988.0020

[31] McMillen L. Job-related tension and anxiety taking a toll among employees in academe's' stress factories. Chronicle of Higher Education. 1987; 33(21):1-9.

[32] Head AJ, Eisenberg MB. Truth be told: How college students evaluate and use information in the digital age [Internet] 2010 [2010 October 20]. Available from: https://ssrn.com/abstract $=2281485$

[33] Barrett A. The information-seeking habits of graduate student researchers in the humanities. Journal of Academic Librarianship. 2005; 31(4):324-31. doi: 10.1016/j.acalib.2005.04.005 
[34] Cummings-Vickaryous B, Mills C, Hoeber L, LeDrew J. Graduate student and instructor engagement in course-based qualitative research: A case study. International Journal for the Scholarship of Teaching and Learning. 2010; 4(2):Article 13. doi: 10.20429/ijsotl.2010.040213

[35] Young S, Jacobs W. Graduate student needs in relation to library research skills. Journal of Modern Education Review. 2013; 3(3):181-91.

[36] Hadjioannou X, Shelton NR, Fu D, Dhanarattigannon J. The road to a doctoral degree: Co-travelers through a perilous passage. College Student Journal. 2007; 41(1):160-177.

[37] Onwuegbuzie AJ, Jiao QG, Bostick SL. Library anxiety: Theory, research, and applications. Lanham, Maryland: Rowman \& Littlefield; 2004.

[38] Einbinder SD. Reducing research anxiety among MSW students. Journal of Teaching in Social Work. 2014; 34(1):2-16. doi: $10.1080 / 08841233.2013 .863263$

[39] Kawakami R. Sources of stress among faculty of higher education [MSc. thesis]. San Jose, California: San Jose State University; 2006.

[40] Saracaloğlu AS. [The relationship between post graduate students' academic motivation level, research anxiety and attitudes with their research competence (Turkish)]. Yüzüncü Yll University Journal of Education Faculty. 2008; 5(2):179-208.

[41] Kracker J. Research anxiety and students' perceptions of research: An experiment. Part I. Effect of teaching Kuhlthau's ISP model. Journal of the American Society for Information Science and Technology. 2002; 53(4):282-94. doi: 10.1002/asi.10040

[42] Trimarco KA. The effects of a graduate learning experience on anxiety, achievement, and expectations in research and statistics. Paper presented at: $28^{\text {th }}$ Annual Conference of the Northeastern Educational Research Association. 1997 October 14; New York USA.

[43] Gmelch WH, Wilke PK, Lovrich NP. Dimensions of stress among university faculty: Factor-analytic results from a national study. Research in Higher Education. 1986; 24(3):266-86. doi: 10.1007/bf00992075

[44] Higgins CC, Kotrlik JW. Factors associated with research anxiety of university human resource education faculty. Career and Technical Education Research. 2006; 31(3):175-99. doi: 10.5328/ cter31.3.175

[45] Gal M, Borg W, Gal J. Quantitative and qualitative research methods in psychology [A. Nasr et al. Persian trans].Tehran: Samt Publication; 2012.

[46] Sahebi A. [Research methods in clinical psychology (Persian)]. Tehran: Samt; 2003.

[47] Sarayi H. [An Introduction to survey sampling (Persian)]. Tehran: Samt; 1993.

[48] Farokhi S, Shareh H. [Psychometric properties of the Persian version of the sexual self-esteem index for woman-short form (Persian)]. Iranian Journal of Psychiatry \& Clinical Psychology. 2014; 20(3):252-63.

[49] Sharifi daramadi P, Qassemi Davari L. [Comparison of emotional intelligence, self-esteem, and depression in crime victim and non-victim girls aged between 15-18 in Tehran (2010-2011)
(Persian)]. Psychology of Exceptional Individuals. 2012; 2(7):11532

[50] Khalatbary J. [Evaluating the effectiveness of behavioralcognitive, cognitive, pharmaceutical, and integrating therapies, with reducing symptoms of trait and state anxiety (Persian)]. Pajoohesh-ha-ye Tarbiati. 2006; 2(2):21-58

[51] Ismaili M. [A survey of the influence of Murita therapy on reducing the rate of anxiety in clients of counseling centers (Persian)]. Research in Clinical Psychology and Counseling. 2011; 1(1):15-30.

[52] Sadeghi S. [Standardization Spielberger test (Persian)] [MA thesis]. Tehran: Islamic Azad University; 2002.

[53] Mahram B. [Spielberger test standardization in Mashhad (Persian)]. [MA thesis]. Tehran: Allameh Tabatabaei University; 1995.

[54] Panahi-shahri M. [Spielberger State-Trait test standardization among students in Isfahan (Persian)] [MA thesis].Tehran: TarbiatModares University; 1994

[55] Marteau TM, Bekker H. The development of a six-item shortform of the state scale of the Spielberger State-Trait Anxiety Inventory (STAI). British Journal of Clinical Psychology. 1992; 31(3):301-6. doi: 10.1111/j.2044-8260.1992.tb00997.x

[56] Spielberger CD, Gorsuch RL. State-trait anxiety inventory for adults: Manual, instrument, and scoring guide: California: Mind Garden, Inc.; 1983.

[57] Büyüköztürk Ş. [Development of anxiety scale for research (Turkish)]. Education Management in Theory and Practice. 1997; 3(4):453-64.

[58] Masoudnia A. [A survey of relationship between self-esteem and social anxiety among undergraduate students (Persian)] Biannual Journal of Clinical Psychology \& Personality. 2009; 16(37):49-58.

[59] Kheirkhah M, Mokarie H, Nisani Samani L, Hosseini AF. [Relationship between anxiety and self-concept in female adolescents (Persian)]. Iran Journal of Nursing. 2013; 26(83):19-29.

[60] Ferro MA, Boyle MH. The impact of chronic physical illness, maternal depressive symptoms, family functioning, and self-esteem on symptoms of anxiety and depression in children. Journal of Abnormal Child Psychology. 2014; 43(1):177-87. doi: 10.1007/ s10802-014-9893-6

[61] Van Tuijl LA, de Jong PJ, Sportel BE, de Hullu E, Nauta MH Implicit and explicit self-esteem and their reciprocal relationship with symptoms of depression and social anxiety: A longitudinal study in adolescents. Journal of Behavior Therapy and Experimental Psychiatry. 2014; 45(1):113-21. doi: 10.1016/j. jbtep.2013.09.007

[62] Yousaf S. The Relation between self-Esteem, parenting style and social anxiety in girls. Journal of Education and Practice. 2015 6(1):140-2.

[63] Khadivi SH, Abedi M, Shabani A. [A study of library and electronic sources anxiety among the students at the university of Isfahan (Persian)]. Library and Information Research Journal. 2009; 8(3):117-134. doi: 10.22067/5721

[64] Mohamdpour Yazdi A, Birashk B, Fata L, Dejkam M. [Casecontrol study of defense styles and state-trait anxiety among college students with general anxiety disorder (Persian)]. Journal of Fundamentals of Mental Health. 2009; 1(11):7-14 
[65] Abdollahian E, Toozandehjani H. [Comparative efficacy of different procedured in reduction of generalized anxiety disorders (Persian)]. Iranian Journal of Psychiatry \& Clinical Psychology. 1994; 1(1):47-57.

[66] Najarian B. [The relationship between test anxiety, general anxiety, self-esteem, teacher expectations and socioeconomic status of students (Persian)]. Journal of Humanities. 1997; 21:17-30. 